\title{
How can optical coherence tomography be used to optimize percutaneous coronary intervention?
}

\author{
Benoit Guillon $^{1,2}$, Hélène Tauzin ${ }^{1}$, Fiona Ecarnot ${ }^{1,2}$, Nicolas Meneveau ${ }^{1,2}$ \\ ${ }^{1}$ Department of Cardiology, University Hospital Besançon, Besançon, France; ${ }^{2}$ EA3920, University of Franche-Comté, Besançon, France \\ Contributions: (I) Conception \& design: B Guillon, N Meneveau; (II) Administrative support: N Meneveau, F Ecarnot; (III) Provision of study \\ materials or patients: N Meneveau, H Tauzin, B Guillon; (IV) Collection and assembly of data: H Tauzin; (V) Data analysis and interpretation: All \\ authors; (VI) Manuscript writing: All authors; (VII) Final approval of manuscript: All authors. \\ Correspondence to: Nicolas Meneveau, MD, PhD. Department of Cardiology, University Hospital Jean Minjoz, Boulevard Fleming, 25000 Besancon, \\ France. Email: Nicolas.meneveau@univ-fcomte.fr.
}

\begin{abstract}
Optical coherence tomography (OCT) imaging provides high resolution assessment of coronary parietal and endoluminal abnormalities. Clinical evidence and intuitive utilization make this tool relevant for use in daily practice in the catheterization laboratory. In the present review, we summarize the benefits of OCT for the optimization of percutaneous coronary intervention (PCI) in daily practice. First, we focus on the characterization of lesion type with a view to anticipating challenges during PCI. Then, we describe the utility of OCT to identify culprit lesions and ambiguous angiographic findings. Finally, we outline targets for optimization after PCI and the mechanisms underlying stent failure.
\end{abstract}

Keywords: Optical coherence tomography (OCT); percutaneous coronary intervention (PCI); underexpansion; malapposition; protrusion; plaque burden; edge dissection; lipid rich pool

Submitted Feb 10, 2020. Accepted for publication Mar 31, 2020.

doi: $10.21037 / \mathrm{cdt} .2020 .04 .02$

View this article at: http://dx.doi.org/10.21037/cdt.2020.04.02

\section{Introduction}

Optical coherence tomography (OCT) is an endocoronary imaging technique that creates high resolution images of the coronary lumen using near-infrared light technology. With its high resolution and intuitive interpretation, OCT has become an essential item of equipment in the interventional catheterization laboratory (cathlab). Image acquisition with automatic pullback is possible in less than 3 seconds, and yields images with a resolution 10 times greater than that of IVUS $(10-20 \mu \mathrm{m})$, although OCT has lower tissue penetration $(1.5 \mathrm{~mm})$ than IVUS $(4-8 \mathrm{~mm})$. Automatic pullback with angiographic coregistration is necessary to ensure accurate interpretation of OCT images, particularly in the context of angioplasty and stent implantation.

Based on registry data, randomized clinical trials and meta-analyses, OCT has proven its efficacy for the improvement of clinical and functional outcomes after percutaneous coronary intervention (PCI) $(1,2)$. OCT- guided PCI is currently recommended by the guidelines for myocardial revascularization of the European Society of Cardiology (ESC) with a grade IIaB recommendation, for the optimization of PCI in selected patients, and to identify mechanisms responsible for stent restenosis (with a grade $\mathrm{IIaC}$ recommendation) (3). In the present review, we summarize the benefits of OCT for the optimization of PCI in daily practice. First, we focus on the characterization of lesion type with a view to anticipating challenges during PCI. Then, we describe the utility of OCT to identify culprit lesions and ambiguous angiographic findings. Finally, we outline targets for optimization after PCI and the mechanisms underlying stent failure.

\section{Planning PCI by OCT}

Identify what type of lesion you are going to treat

OCT is a useful complement to compensate for the pitfalls 
Table 1 Plaque features assessed by OCT and associated with poor outcomes

Lipid rich pool at the edge of the stent
Lipid arc circumference $>180^{\circ}$
Calcium $\operatorname{arc}>180^{\circ}$ and thickness $>0.5 \mathrm{~mm}$
MLA $<3.5 \mathrm{~mm}^{2}(\mathrm{LAD})$
Macrophages
Thin-cap atheroma $<75 \mu \mathrm{m}$
OCT, optical coherence tomography; MLA, minimal lumen area;
LAD, left anterior descending artery.

of angiography in assessing the parietal components of coronary vessels, such as calcifications and plaque composition. OCT makes it possible to quantify total calcium arc and calcium thickness, and calcium arc $>180^{\circ}$ and thickness $>0.5 \mathrm{~mm}$ have been shown to predict stent under-expansion (4). These thresholds have been suggested as criteria for the use of debulking techniques such as rotational atherectomy or intravascular lithotripsy for the purposes of lesion preparation $(4,5)$. Conversely, lipidrich plaque in the stent landing zone is associated with more frequent no-reflow and type 4 myocardial infarction $(6,7)$, and should be avoided. Considering a lesion without angiographic evidence of ischemic potential, the CLIMA study investigated the individual and pooled impact of OCT-defined plaque vulnerability features on 1 year clinical outcomes. Taken together, thin cap atheroma $<75 \mu \mathrm{m}$, lipid arc circumferential extension $>180^{\circ}$, MLA $<3.5 \mathrm{~mm}^{2}$ and OCT defined macrophages were associated with an increased risk of cardiac death or target segment MI (HR 7.54, 95\% CI: 3.1-18.6) (8). Plaque features associated with poor outcomes are listed in Table 1.

\section{Plaque rupture}

Plaque rupture is mostly found in ST segment elevation MI (STEMI) (70\% vs. $55 \%$ in non-STEMI) (9). OCT shows loss of intimal integrity with a cavity in the arterial wall (Figure 1), and thrombus is frequently present. Predictors of plaque rupture are thin cap fibro-atheroma (TCFA) and hypertension (10). Compared to plaque erosion, plaque rupture is associated poorer angiographic findings, such as more severe stenosis, more TIMI 0 flow, and more distal embolization $(10,11)$. However, reported findings in terms of adverse outcomes are conflicting, with no difference between plaque rupture and plaque erosion in some reports $(10,11)$, whereas Niccoli et al. found significantly more major adverse cardiac and cerebrovascular events (MACCE) in case of plaque rupture (12). There are few hypotheses in support of this observation: firstly, higher unstable atherosclerosis features, secondly, a higher risk of stent failure including stent thrombosis possibly related to delayed endothelialization and residual pro-thrombotic material (13).

\section{Plaque erosion}

Angiographic findings are mostly normal and OCT hallmarks are the presence of thrombus, and an intact plaque (Figure 2).

Plaque erosion underpins a quarter of all ACS, predominantly NSTE-ACS. Contrary to plaque rupture, patients with plaque erosion are younger, more often female and more often smokers (9). Significant stenosis is less frequent than in the case of plaque rupture, leading to fewer angiographic abnormalities with plaque erosion (11). In addition, registries have shown that plaque erosion is not associated with TCFA and lipid rich pool $(10,11)$. OCT plaque erosion diagnosis is a key step of investigation during the work-up of MI with normal coronary arteries (MINOCA). Indeed, the EROSION study suggested that a conservative antithrombotic strategy was safe in case of plaque erosion without stenosis $>70 \%$, with a low rate of MACE at 1 year (5.7\%) and absence of any residual thrombus in $46.9 \%$ at one year (14).

\section{Eruptive calcified nodules}

Calcified nodules are the third leading mechanism of atherothrombosis (15). Sometimes angiographic findings are unable to differentiate calcified nodules from thrombus. Calcifications involve high attenuation with regular outline. Conversely, lipid pool outlines are blurry. Calcified nodules often protrude into the vessel lumen with high signal attenuation (Figure 3). As indicated above, a calcification arc $>180^{\circ}$ and $>0.5 \mathrm{~mm}$ thickness is associated with stent underexpansion $(4,5)$ and thus, have potential prognostic value when identified before angioplasty.

\section{Spontaneous coronary artery dissection (SCAD) and coronary hematomas}

OCT has significantly contributed to improving knowledge of the pathophysiology and diagnosis of SCAD. Thanks to its high resolution, OCT is able to identify intramural hematoma induced by disruption of the media layer (Figure 4). A statement from the American Heart Association (AHA) identifies 3 types of SCAD characterized by different 


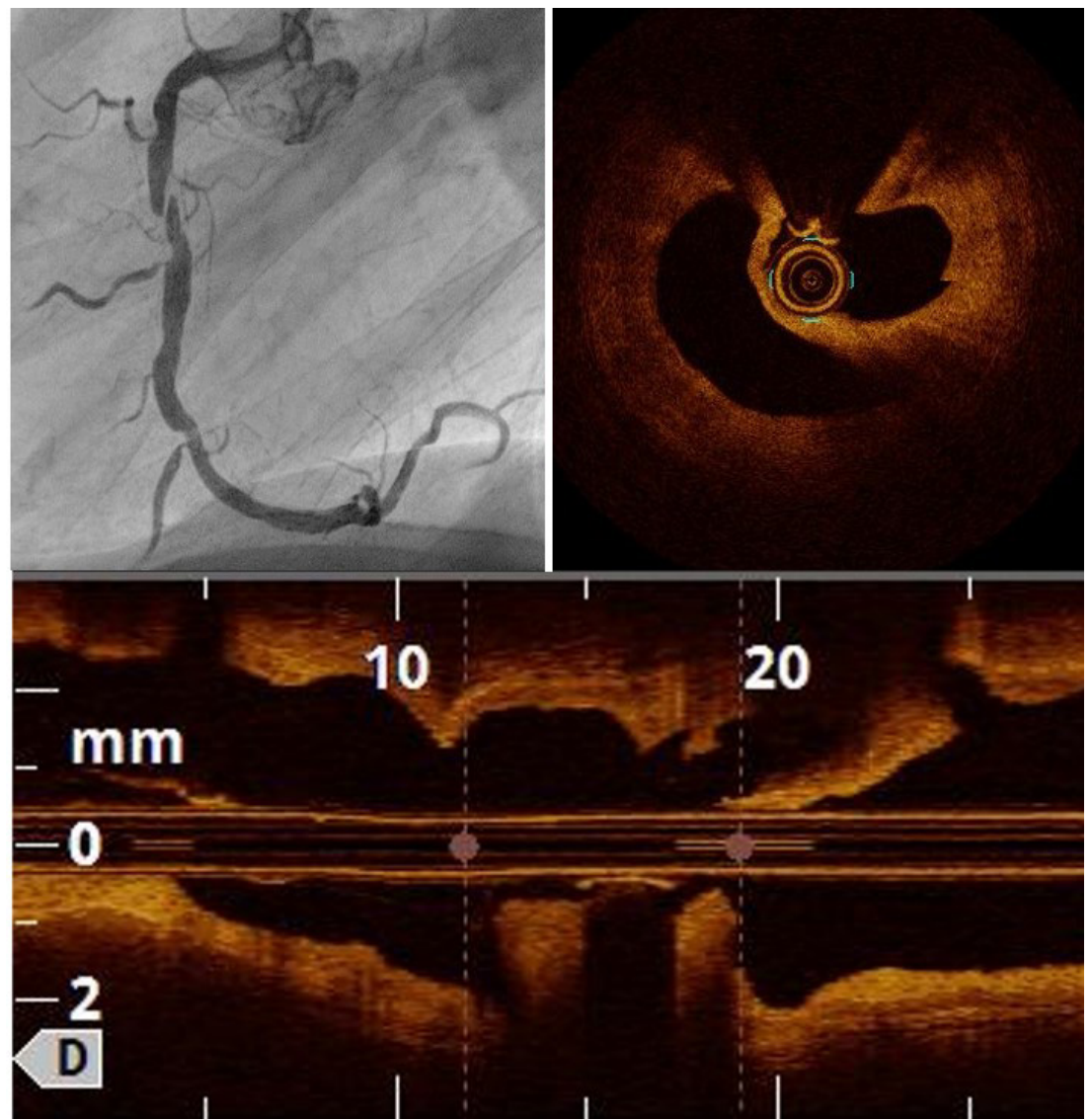

Figure 1 Plaque rupture: angiography and OCT features. Angiographic mild RCA stenosis with initial suspicion of spontaneous coronary dissection (right). OCT shows pseudo dual lumen corresponding to a non-thrombotic chronic plaque rupture (left and bottom). OCT, optical coherence tomography.

angiographic features (16). The first type is easy to identify with angiography as a wall stain or flap. The second type corresponds to diffuse, smooth stenosis. The third type mimics a focal atherosclerotic lesion. OCT imaging should be limited to non-informative angiographic features. Indeed, the use of a guide wire and contrast medium flush incur a risk of worsening hematoma or dissection extension to total occlusion. For this reason, the risk associated with OCT imaging should systematically be weighed against the potential benefit. In conclusion, OCT provides determinant information in the diagnosis of type $3 \mathrm{SCAD}$, and can make it possible to avoid futile and potentially harmful PCI $(16,17)$. The natural history of SCAD is mostly favorable with conservative management and spontaneous healing (16). Nonetheless, aggressive therapy including cutting balloon, stenting or surgery should be considered in case of persistent ischaemia or haemodynamic instability.

\section{Size the stent accordingly}

Selecting the right stent diameter is key to avoiding complications. The consensus statement from the EAPCI (5) recommends selecting the stent diameter based on the distal lumen reference diameter, rounding up to the next available stent diameter.

The measurement of the proximal and distal references used to guide the choice of stent diameter depends on whether the external elastic lamina (EEL) is visible or not. If the EEL is visible, the measure of the reference diameters should be the average of the distance from EEL to EEL in two orthogonal views. In this case, the reference should be rounded down to the nearest available stent size. If the EEL is not visible, the average lumen diameter should be used, as 

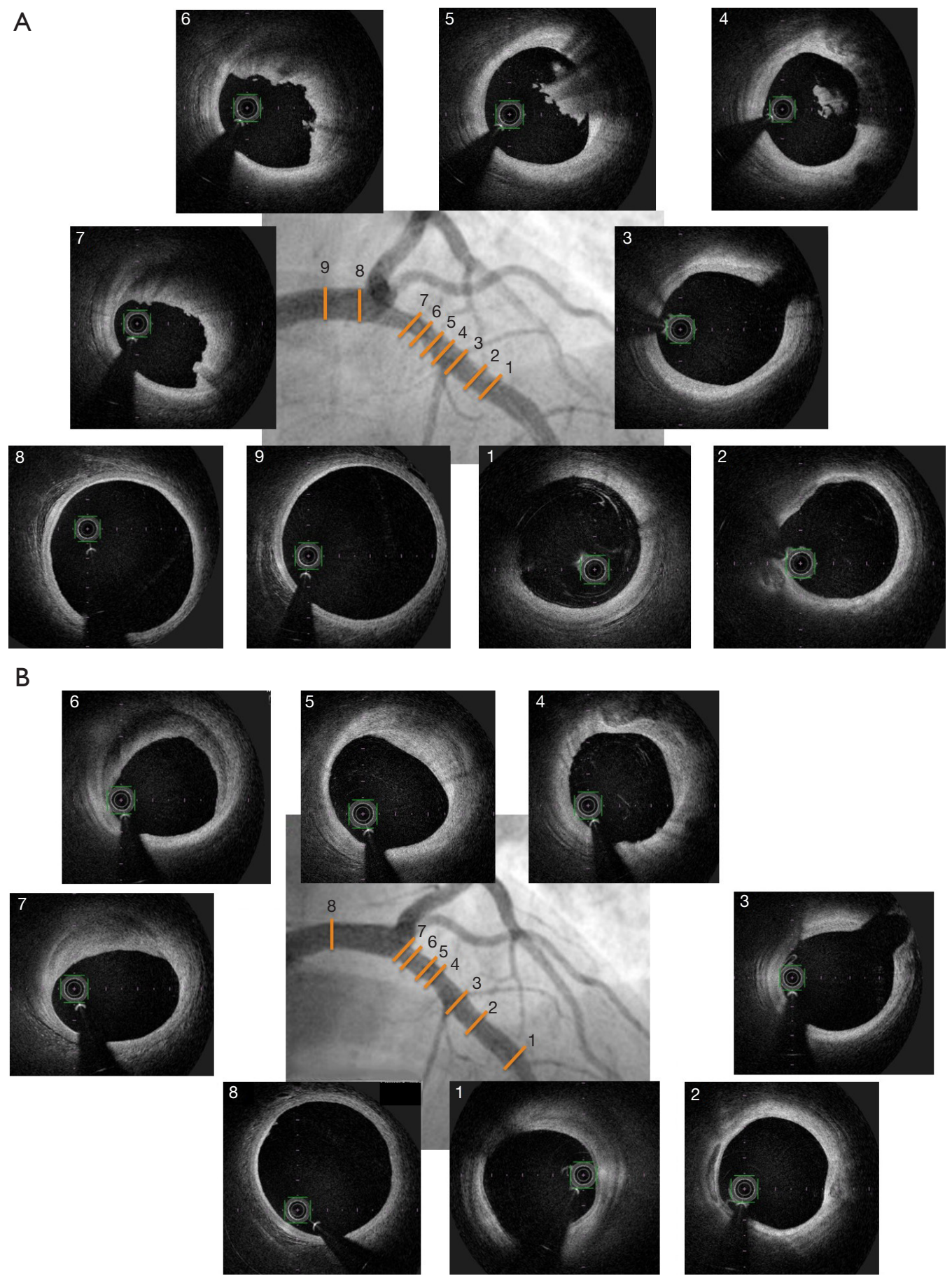

Figure 2 Angiographic and OFDI features of a LAD plaque erosion. LAD plaque erosion (A) responsible for NSTE-ACS at the acute phase and (B) 1 month later. We observed total regression of thrombotic burden underlying fibro-calcic plaque. OFDI, optical frequency domain imaging; LAD, left anterior descending artery; ACS, acute coronary syndrome. 

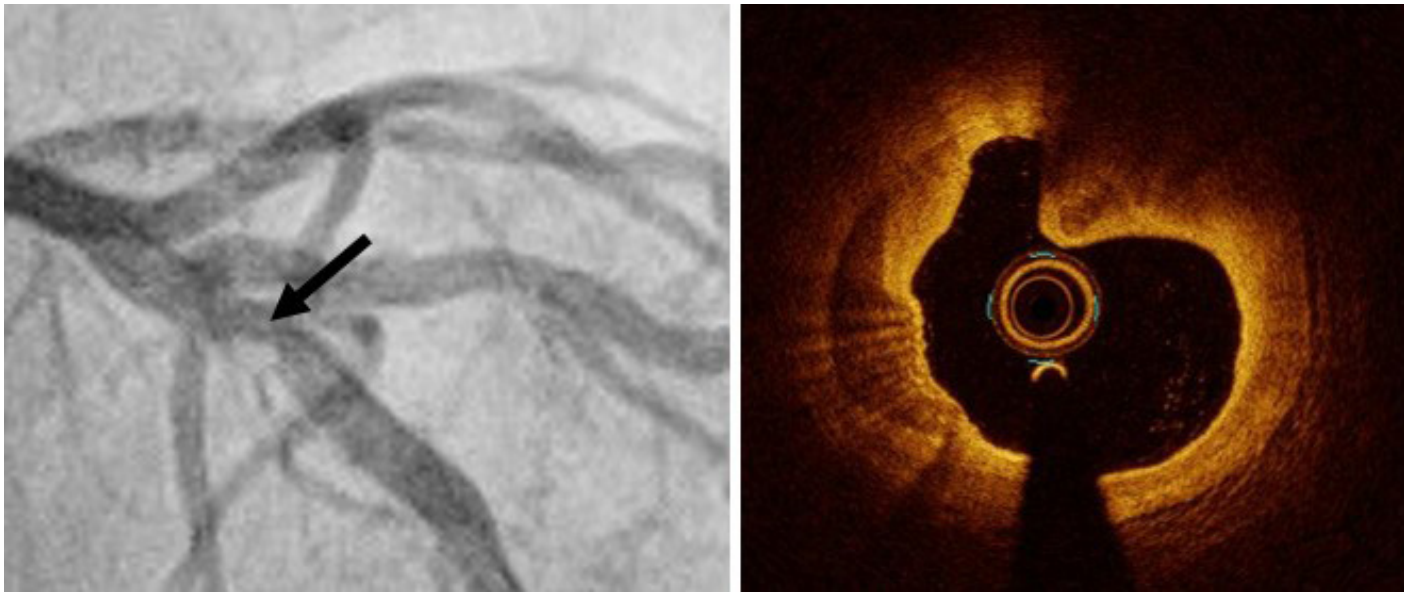

Figure 3 Eruptive calcic nodule: angiography and OCT features. Ambiguous angiographic features between thrombus and calcification are resolved by OCT. Typical OCT findings are ab-luminal high attenuation with regular outline. OCT, optical coherence tomography.

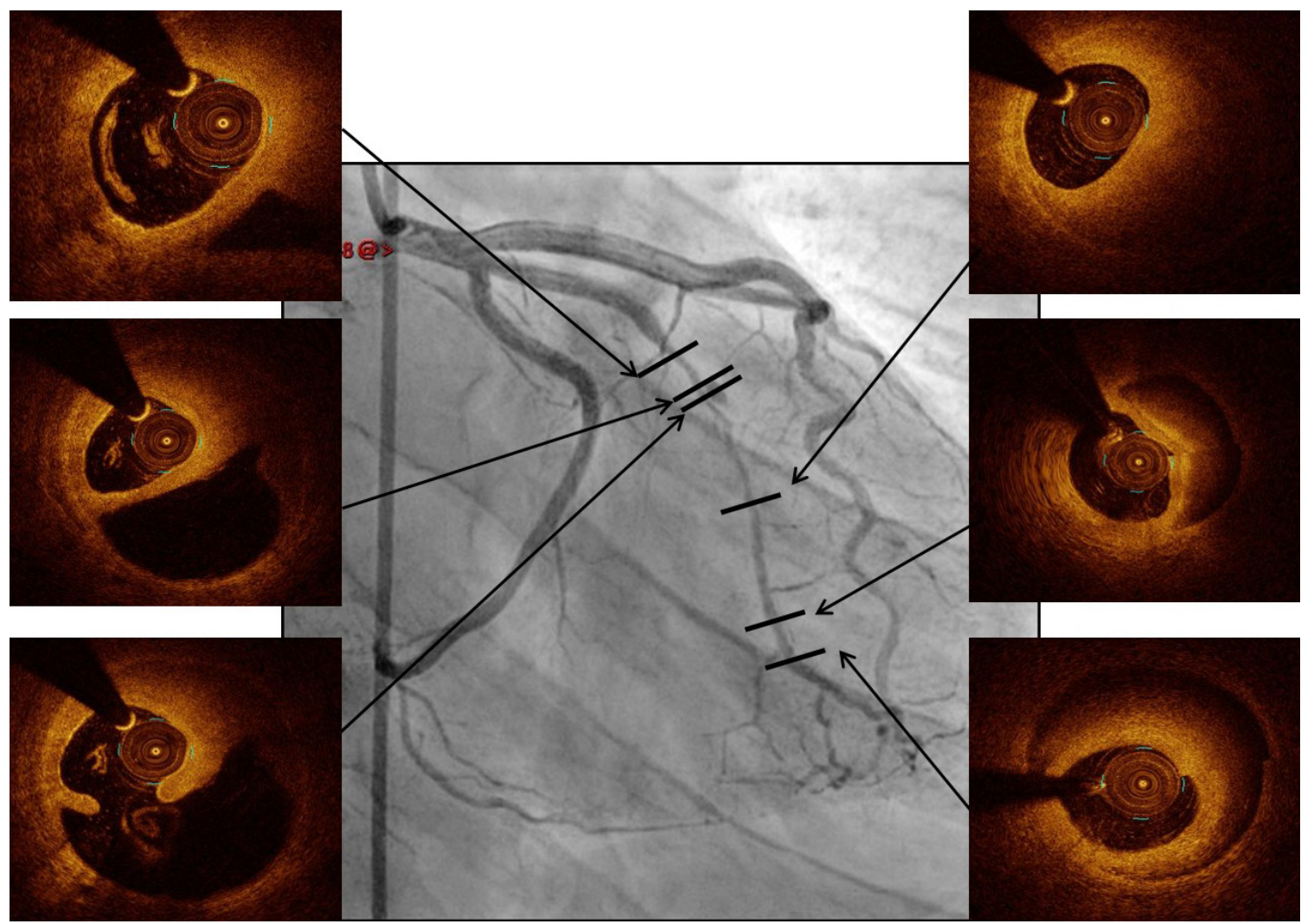

Figure 4 Angiographic and OCT features of spontaneous coronary dissection. Spontaneous type 1 first marginal artery dissection involved in lateral STE-ACS. Right pictures show entry of the intimal tears then flap intimal with true and false lumen. Left pictures show thrombosis of the wall hematoma. OCT, optical coherence tomography; ACS, acute coronary syndrome. 

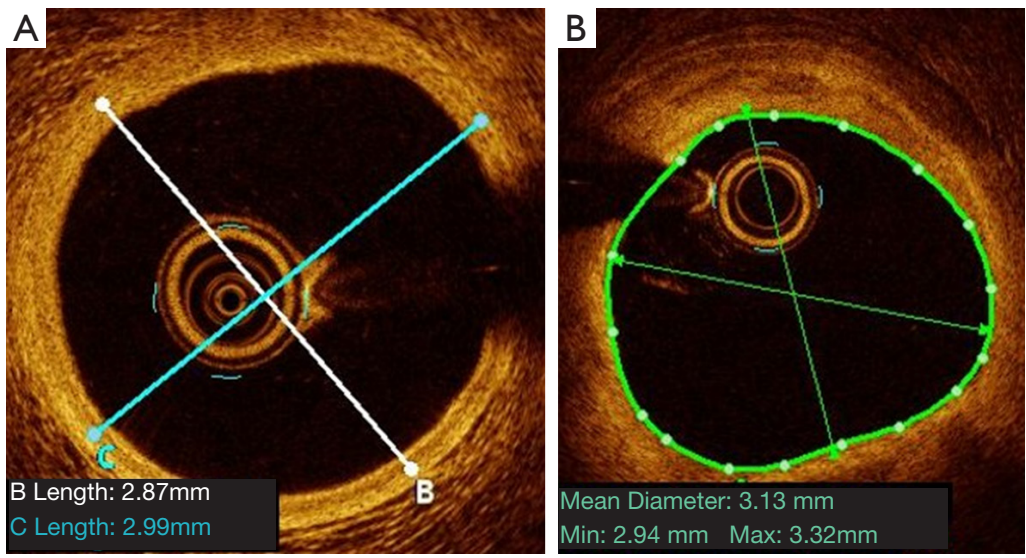

Figure 5 Stent sizing by OCT. (A) Picture shows average external elastic membrane diameter in two orthogonal measurements and (B) picture shows average luminal diameter in two orthogonal measurement. OCT, optical coherence tomography.

calculated from two orthogonal views, and the value should be rounded up to the nearest available stent size (Figure 5) (as per the criteria used in the ILUMIEN IV study, NCT03507777). This is particularly important in case of long lesions and bifurcations, where diameter disparity is high, due to Finet's law (18).

Stent length should be based on optimal lesion coverage. It implies that the landing zone should be situated in healthy vessel or where there is low atherosclerotic burden. Previous data from studies with intravascular ultrasound (IVUS) have shown that residual plaque burden $>70 \%$ after PCI was associated with a significantly higher rate of major adverse cardiac events (MACE) (19). In addition, unpublished data from the DOCTORS study show that residual plaque burden $>50 \%$ at the edge of the stent is associated with lower FFR values post-stenting (20). As mentioned above, a landing zone on lipid-rich plaque should be avoided.

\section{Optimize PCI with OCT, using recommended targets}

Six precise criteria (Figure 6) have been listed by the EAPCI consensus statement as features to be focused on in the context of OCT-guided PCI (5). While this consensus statement clearly defines OCT features and their justification, it should be noted that the level of evidence is often low, with discordant results between existing studies.

\section{Optimal stent expansion}

Optimal stent expansion corresponds to ability of the stent to achieve a goal of intrastent minimum surface area. Thus, optimal stent expansion is assessed either by the absolute minimum stent area, or by the ratio of minimum stent area to reference vessel area (Figure 7).

A minimal stent area (MSA) of $>5.44 \mathrm{~mm}^{2}$ was found to be a predictor of FFR $>0.9$ in the DOCTORS study (2). The CLI-OPCI study showed that a MSA $<4.5 \mathrm{~mm}^{2}$ was an independent predictor of MACE after PCI (21). Nonetheless, the assessment of stent expansion could be challenged in case of very large vessels $(>5.5 \mathrm{~mm})$ such as the left main, by limited OCT penetration.

For this reason, the EAPCI consensus statement recommends optimizing stent expansion using the ratio of $\mathrm{MSA} /$ average reference surface area, with a goal of $>80 \%$. This $80 \%$ cut-off was chosen based on the impact of OCTguided vs. angio-guided PCI on the final FFR value in the DOCTORS study (2). Moreover, stent thrombosis registries have shown that stent under-expansion was significantly associated with acute and sub-acute stent thrombosis $(22,23)$. Indeed, it has been shown that $50 \%$ to $60 \%$ of patients have sub-optimal post-PCI FFR (defined as FFR <0.90), mainly due to stent under-expansion $(2,24)$. Stent optimization is possible using pre and/or post stenting balloon inflation, and if necessary, endovascular therapy can be envisaged to treat calcified lesions (e.g., Rotablator). The ongoing ILUMIEN IV study (NCT03507777) is assessing the potential benefit of OCT-guided procedures in long lesions $(>28 \mathrm{~mm})$ and may provide new insights in this area.

\section{Malapposition}

Malapposition corresponds to a lack of contact between 


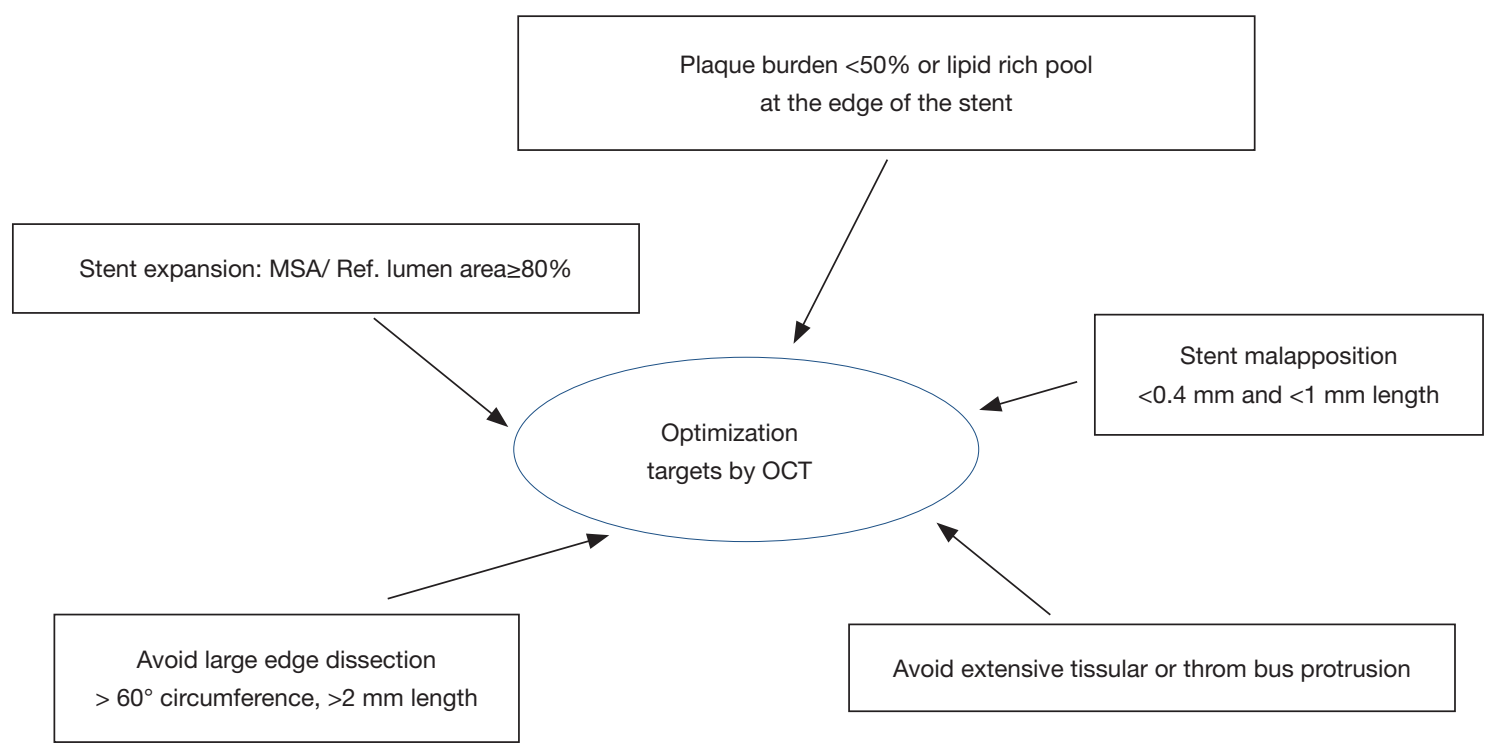

Figure 6 Criteria to achieve for optimal stenting guided by OCT. OCT, optical coherence tomography.

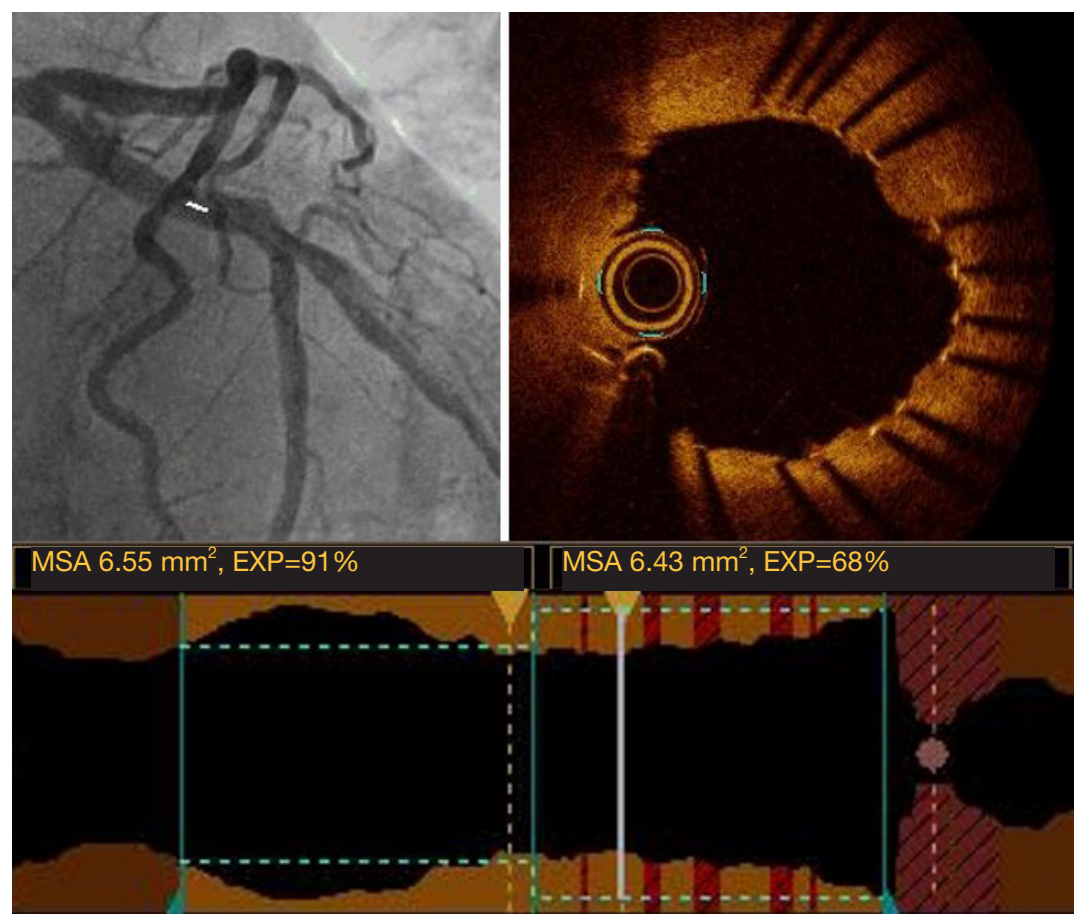

Figure 7 Circumflex artery stent under expansion assessed by OCT. Minimal sent area/reference lumen area $=68 \%$ corresponding to a significant under expansion. OCT, optical coherence tomography.

the struts of the stent and the intima layer of the vessel at the acute phase of stent implantation (Figure 8). Im et al. identified calcified lesions, stent length $>25 \mathrm{~mm}$ and baseline diameter stenosis $>70 \%$ as predictors of acute stent malapposition (25). Moreover, the risk of severe malapposition is increased in case of bifurcation lesions (5).

Prospective data reporting the negative impact of malapposition are scarce. Data from registries suggest that 

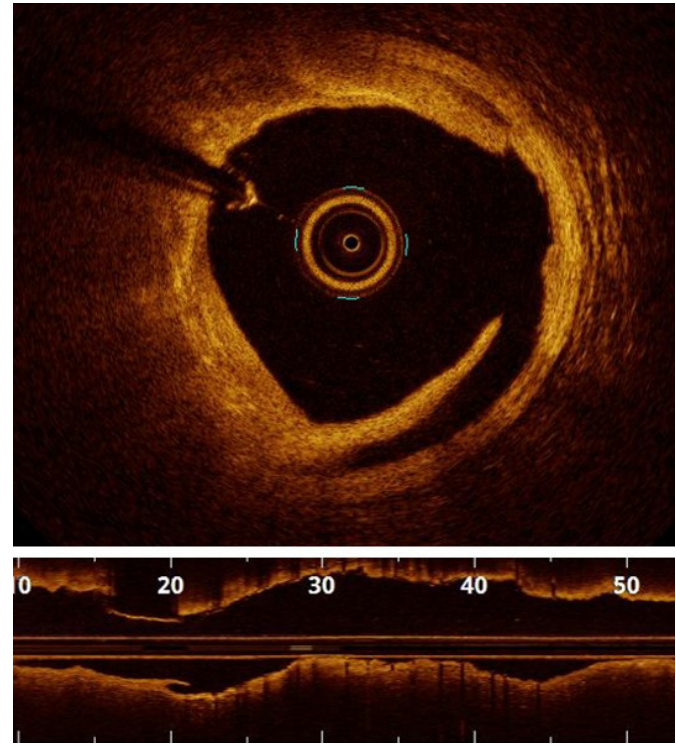

Figure 8 Struts malapposition detected by OCT. Axial distance from struts to intima is $0.73 \mathrm{~mm}$. OCT, optical coherence tomography.

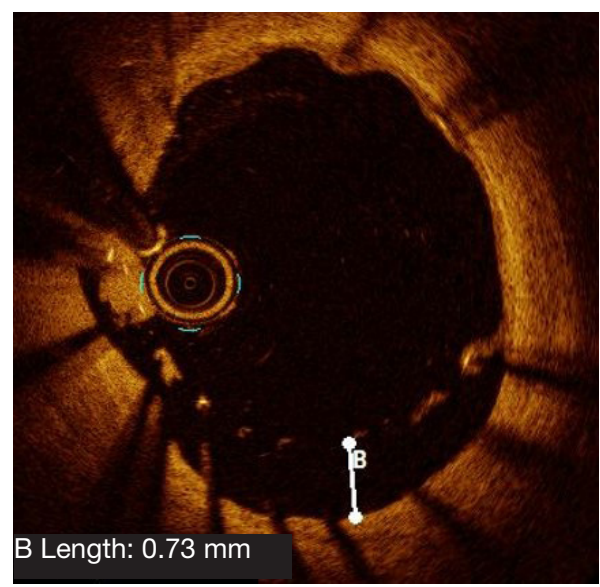

Figure 9 Thrombus protrusion in OCT. Thrombus is protruding into the struts of the stent implanted to open an occluded right coronary artery. OCT, optical coherence tomography.

acute malapposition is associated with early and non-early stent thrombosis $(22,23)$. The EAPCI consensus stipulates that an axial distance between struts and intima $<0.4 \mathrm{~mm}$ with length $<1 \mathrm{~mm}$ is an acceptable level of malapposition. Malapposition can be improved by post-stent balloon inflation taking into account reference lumen diameter. In this context, it should be kept in mind that a balloon can never adapt to a non-circular vessel shape, e.g., elliptic

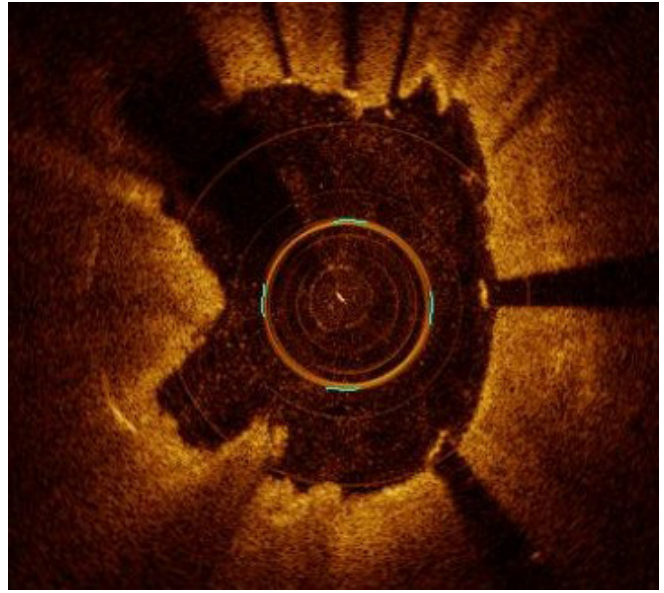

Figure 10 Dissection visualized by OCT. Distal intimal edge stent dissection which should be treated by additional stenting. OCT, optical coherence tomography.

arteries or the LM. The latest software packages now enable automatic identification of malapposed stent struts (OCT Abbott $^{\circledR}$ ILUMIEN OPTIS 3D software update, Aptivue ${ }^{\circledR}$ ).

\section{Tissue protrusion}

Tissue protrusion corresponds to the presence of plaque material or thrombus bulging through the struts of the stent into the lumen (Figure 9). Reports of its prognostic impact on clinical events are discordant. No adverse effect on clinical outcomes was observed in case of tissue prolapse observed on IVUS in a study by Qiu et al. (26). In a 1-year follow-up study of a multicenter registry, Soeda et al. (27) characterized protrusion findings immediately after stent implantation in three types, namely smooth, disrupted fibrous tissue and irregular protrusion. This definition was based on intima integrity below the struts. Irregular protrusion was the only protrusion criterion associated with device-oriented cardiac events (27). The EAPCI consensus statement recognizes the presence of tissue or thrombus prolapse as a marker of poor prognosis in case of elective or urgent PCI (5).

\section{Edge dissection}

Edge dissection corresponds to intima rupture at the proximal and/or distal part of the stent (Figure 10). Distal dissection was found to be a risk factor for MACE in the CLiOPCI 2 study (21). In this study, edge dissection was 


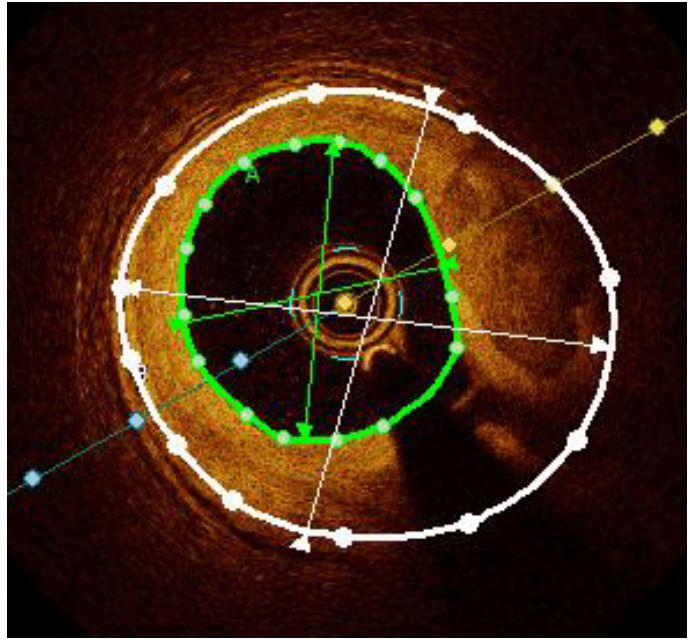

Figure 11 Plaque burden assessment by OCT. Calcified plaque burden is calculated at $62 \%$ at the distal edge of the stent. OCT, optical coherence tomography.

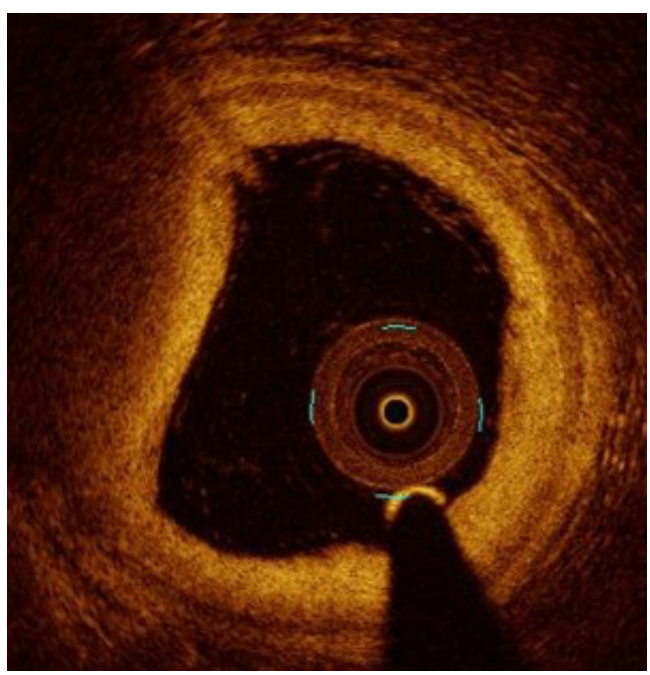

Figure 12 Hemi-circumferential lipid rich pool in OCT. Lipid plaque has high attenuation of the signal with blur outlines. OCT, optical coherence tomography.

defined as a rim of tissue with a width $\geq 200 \mu \mathrm{m}$ and a clear separation from the vessel wall. Certain registries found that edge dissection was observed in case of acute stent thrombosis without significance $(22,23)$. Independent predictors of edge dissection include presence of plaque at the landing zone, presence of TCFA and stent overexpansion (28). The EAPCI consensus does not recommend additional intervention in case of edge dissection with an arc $<60^{\circ}$, measuring $<2 \mathrm{~mm}$ length and limited to the intima.

\section{Plaque burden $>50 \%$}

Plaque burden means a burden of atherosclerotic components at the edge of the stenting zone. It reflects concentric and eccentric atherosclerotic remodeling of the vessel.

Plaque burden is calculated using the vessel surface area (VSA), as delimited by the internal elastic membrane (IEM), and the lumen surface area (LSA), as defined by intimal area (Figure 11).

$$
\text { Plaque burden }(\%)=\frac{V S A-L S A}{V S A} \times 100
$$

Up to present, OCT has not been widely used to measure plaque burden due to its low penetration into the layers of the vessel. However, data have shown that plaque burden measured by IVUS was associated with a higher rate of stent restenosis $(19,29)$. Recent unpublished data from the DOCTORS study (20) showed the feasibility of assessing plaque burden by OCT, and the functional impact of plaque burden $>50 \%$ after PCI in the context of ACS. Plaque burden was found to be a major predictor of lower final FFR. Plaque burden $<50 \%$ is a target of optimal PCI according to the EAPCI.

\section{Lipid rich pool}

Lipid rich pool is visualized on OCT as high attenuation parietal plaque with blurred outlines (Figure 12). The EAPCI consensus identified lipid rich pool plaque as an unfavorable feature that should be avoided after PCI, due to an excess risk of no-reflow phenomenon, especially when the lipid arc is high (6). Consequently, lipid-rich pool in the stent landing zone should be avoided, where possible, or covered entirely by the stent.

\section{Optimizing PCI in special populations: bifurcation and LM lesions}

The role of OCT in bifurcation lesions, especially lesions of the LM, is twofold. First, OCT is helpful in choosing the optimal stent diameter. Selection of the stent diameter has to be based on the distal main vessel. The proximal main branch will be overexpanded by balloon inflation during proximal optimization technique (POT). Thus, OCT measurements give the distal stent diameter and 

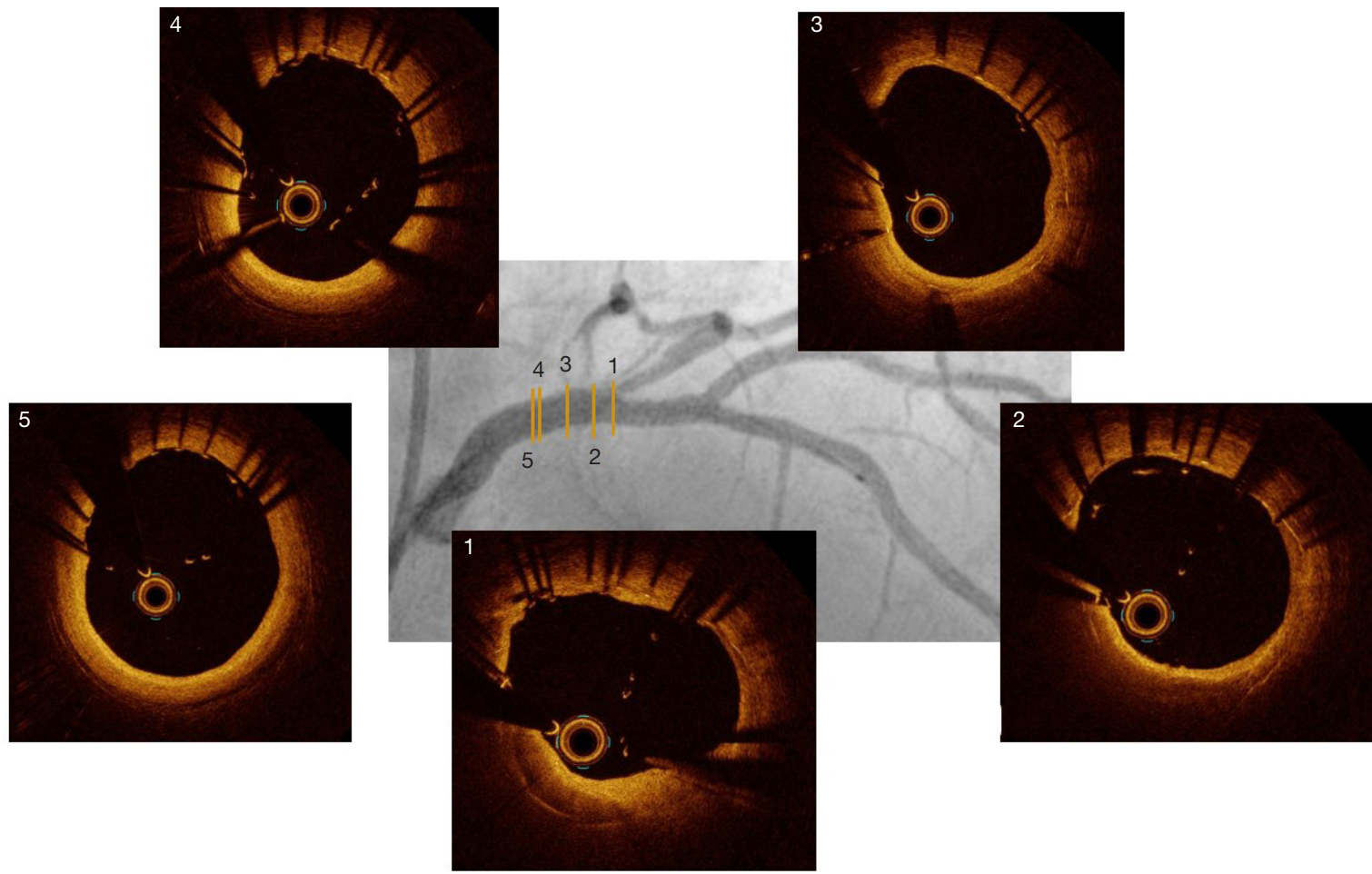

Figure 13 Contribution of OCT to bifurcation treatment by PCI. Severe stent malapposition induced by accidental recrossing of the wire of the circumflex artery side branch after LMA/LAD stenting. Guidewire is outside the stent. OCT, optical coherence tomography; PCI, percutaneous coronary intervention; LAD, left anterior descending artery.

proximal balloon diameter. Of course, OCT also provides details about optimal stenting (e.g., malapposition, underexpansion).

Secondly, OCT helps to avoid abnormal (outside of the stent) recrossing of guidewires in the side-branch in case of provisional POT stenting, TAP or culotte. Extra-stent recrossing (between the struts and the arterial wall) leads to stent failure by stent crushing (Figure 13). Moreover, it has been demonstrated that recrossing the side branch in the distal stent cell was associated with better strut apposition at the side branch ostium (30). Three-dimensional computation by OCT helps to identify right cell recrossing.

In view of the impact of PCI failure (restenosis and stent thrombosis) on the left main stem, and the fact that PCI of the LM is always a bifurcation approach involving one or two stents, we suggest that OCT should always be used for PCI of the left main.

Given the need for optimal clearance of contrast medium to obtain high quality imaging and engagement of the guiding catheter, ostial LM disease remains a challenge with OCT that IVUS is able to overcome. Moreover, large vessels (more than $>5 \mathrm{~mm}$ diameter) are also challenging for OCT because of its limited penetration. Optimal apposition of the stent is sometimes unfeasible because of the elliptic shape of the LM, which a circular balloon can never improve. Thus, we have to keep in mind that excessive search of OCT perfection can lead to procedural complications. Despite a slightly higher volume of contrast during OCT-guided procedures, and a higher level of radiation, there was no significant difference in the risk of acute kidney injury between groups in the DOCTORS study (2).

No MLA cut-off on OCT has ever been established and associated with FFR that makes it possible to indicate or contra-indicate revascularization of the LM. An MLA $<4.5 \mathrm{~mm}^{2}(31)$ as assessed by IVUS was shown to be a useful index of an FFR of $\leq 0.80$ by Park et al, but this value cannot be generalized to OCT. A small registry of 120 patients showed that intermediate area stenosis $50-75 \%$ with MLA $\leq 4 \mathrm{~mm}^{2}$ and ulcerated plaque or critical ostial stenosis who underwent revascularization had the same prognosis as patients with $\mathrm{LM}$ area stenosis $<75 \%$ and 

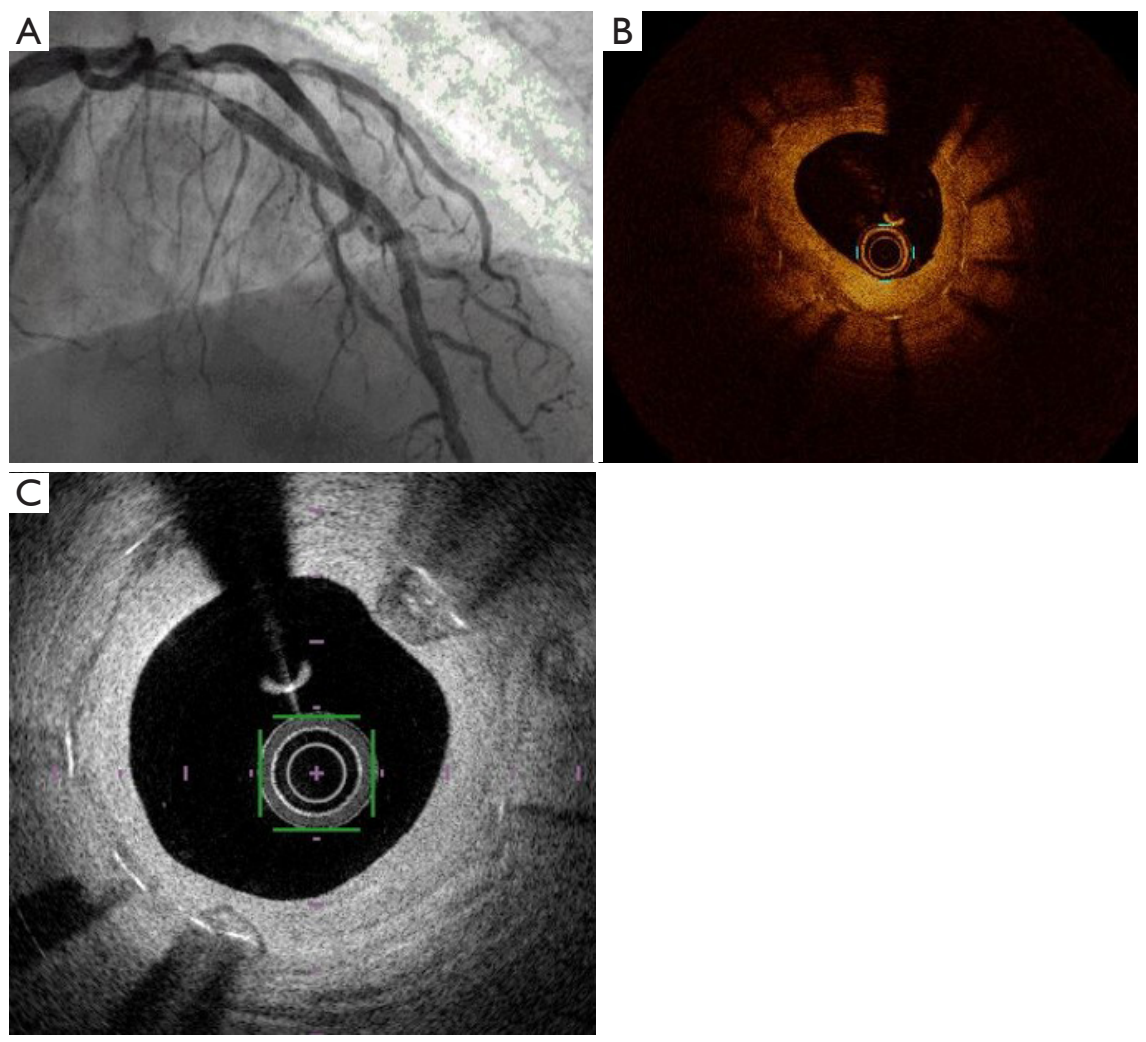

Figure 14 Intra-stent stenosis assessed by OCT. (A) Neointimal hyperplasia after BMS stenting; (B) neo-atherogenesis with calcifications. OCT, optical coherence tomography.

MLA $>4 \mathrm{~mm}^{2}$ treated conservatively (32). Hyperemic functional assessment still remains the gold standard to assess myocardial ischemia (3).

\section{Identify mechanisms of stent failure with OCT to guide PCI strategy}

\section{Restenosis}

OCT is able to differentiate neoatherosclerosis from neointimal hyperplasia, which are the two causes of intrastent stenosis (Figure 14). Neoatherosclerosis has the same components as primitive atherosclerotic lesions, suggesting that it could be treated in the same manner as a de novo lesion, i.e., by additional stent implantation. Neointimal proliferation, on the other hand, could be treated by angioplasty with drug-coated balloon. However, neither of these strategies has been evaluated in randomized studies, and both warrant investigation in dedicated prospective trials.

\section{Stent thrombosis}

Mechanistic causes of stent thrombosis have been described in several stent thrombosis registries like PESTO and PRESTIGE $(22,23)$. The main causes of early stent thrombosis are malapposition, under-expansion and edge dissection. Late stent thrombosis is favored by malapposition, neoatherosclerosis and uncovered struts (no late endothelialization of the implanted stent). Identification of the mechanisms of stent failure is a key step to correcting risk factors of its occurrence, e.g., under-expansion or edge dissection. Edge dissection should be corrected by additional stenting, whereas under-expansion is treatable by balloon overdilation.

Moreover, OCT imaging was able to characterize new phenomena responsible for bioresorbable vascular scaffold thrombosis, called strut discontinuity, corresponding to spontaneous strut dislocation into the lumen despite initially adequate apposition (33). 


\section{Future directions}

Endo-vascular coronary anatomy assessment is very accurate by OCT. Nonetheless, the physiology is not taken into account in terms of results after PCI. OFR (OCT-based optical flow ratio) is a computation of FFR from OCT imaging. New data have shown excellent agreement with FFR (34). It suggests that coronary artery disease evaluation could be performed with anatomy and physiology in order to indicate, plan and optimize PCI.

\section{Conclusions}

OCT is an additional tool in the armamentarium of interventional cardiologists during PCI. Its high resolution and ease of use make it possible to assess endoluminal and wall abnormalities in the coronary arteries. The first step is the identification of the mechanism and type of plaque, in order to define procedural strategy and anticipate challenges during PCI. OCT assessment may also make it possible to avoid unnecessary and potentially harmful PCI. The second step is to assess the results of PCI and optimize the procedure according to published criteria. These are particularly important in case of PCI on a crucial site, such as the left main. Finally, OCT provides insights into the mechanisms of stent failure. Taken together, these considerations make OCT the physician's friend in optimizing PCI.

\section{Acknowledgments}

Funding: None.

\section{Footnote}

Provenance and Peer Review: This article was commissioned by the Guest Editor (Dennis T. L. Wong) for the series "Intracoronary Imaging" published in Cardiovascular Diagnosis and Therapy. The article was sent for external peer review organized by the Guest Editor and the editorial office.

Conflicts of Interest: All authors have completed the ICMJE uniform disclosure form (available at http://dx.doi. org/10.21037/cdt.2020.04.02). The series "Intracoronary Imaging" was commissioned by the editorial office without any funding or sponsorship. NM reports personal fees from Abbott, personal fees from Terumo, outside the submitted work. The other authors have no other conflicts of interest to declare.

Ethical Statement: The authors are accountable for all aspects of the work in ensuring that questions related to the accuracy or integrity of any part of the work are appropriately investigated and resolved.

Open Access Statement: This is an Open Access article distributed in accordance with the Creative Commons Attribution-NonCommercial-NoDerivs 4.0 International License (CC BY-NC-ND 4.0), which permits the noncommercial replication and distribution of the article with the strict proviso that no changes or edits are made and the original work is properly cited (including links to both the formal publication through the relevant DOI and the license). See: https://creativecommons.org/licenses/by-nc-nd/4.0/.

\section{References}

1. Buccheri S, Franchina G, Romano S, et al. Clinical Outcomes Following Intravascular Imaging-Guided Versus Coronary Angiography-Guided Percutaneous Coronary Intervention With Stent Implantation: A Systematic Review and Bayesian Network Meta-Analysis of 31 Studies and 17,882 Patients. JACC Cardiovasc Interv 2017;10:2488-98.

2. Meneveau N, Souteyrand G, Motreff P, et al. Optical Coherence Tomography to Optimize Results of Percutaneous Coronary Intervention in Patients with Non-ST-Elevation Acute Coronary Syndrome: Results of the Multicenter, Randomized DOCTORS Study (Does Optical Coherence Tomography Optimize Results of Stenting). Circulation 2016;134:906-17.

3. Neumann FJ, Sousa-Uva M, Ahlsson A, et al. 2018 ESC/ EACTS Guidelines on myocardial revascularization. Eur Heart J 2019;40:87-165.

4. Fujino A, Mintz GS, Matsumura M, et al. A new optical coherence tomography-based calcium scoring system to predict stent underexpansion. EuroIntervention 2018;13:e2182-9.

5. Raber L, Mintz GS, Koskinas KC, et al. Clinical use of intracoronary imaging. Part 1: guidance and optimization of coronary interventions. An expert consensus document of the European Association of Percutaneous Cardiovascular Interventions. Eur Heart J 2018;39:3281-300.

6. Tanaka A, Imanishi T, Kitabata H, et al. Lipid-rich plaque 
and myocardial perfusion after successful stenting in patients with non-ST-segment elevation acute coronary syndrome: an optical coherence tomography study. Eur Heart J 2009;30:1348-55.

7. Imola F, Occhipinti M, Biondi-Zoccai G, et al. Association between proximal stent edge positioning on atherosclerotic plaques containing lipid pools and postprocedural myocardial infarction (from the CLI-POOL Study). Am J Cardiol 2013;111:526-31.

8. Prati F, Romagnoli E, Gatto L, et al. Relationship between coronary plaque morphology of the left anterior descending artery and 12 months clinical outcome: the CLIMA study. Eur Heart J 2020;41:383-91.

9. Iannaccone M, Quadri G, Taha S, et al. Prevalence and predictors of culprit plaque rupture at OCT in patients with coronary artery disease: a meta-analysis. Eur Heart J Cardiovasc Imaging 2016;17:1128-37.

10. Saia F, Komukai K, Capodanno D, et al. Eroded Versus Ruptured Plaques at the Culprit Site of STEMI: In Vivo Pathophysiological Features and Response to Primary PCI. JACC Cardiovasc Imaging 2015;8:566-75.

11. Hu S, Zhu Y, Zhang Y, et al. Management and Outcome of Patients With Acute Coronary Syndrome Caused by Plaque Rupture Versus Plaque Erosion: An Intravascular Optical Coherence Tomography Study. J Am Heart Assoc 2017;6.

12. Niccoli G, Scalone G, Crea F. Acute myocardial infarction with no obstructive coronary atherosclerosis: mechanisms and management. Eur Heart J 2015;36:475-81.

13. Vergallo R, Ren X, Yonetsu T, et al. Pancoronary plaque vulnerability in patients with acute coronary syndrome and ruptured culprit plaque: a 3-vessel optical coherence tomography study. Am Heart J 2014;167:59-67.

14. Xing L, Yamamoto E, Sugiyama T, et al. EROSION Study (Effective Anti-Thrombotic Therapy Without Stenting: Intravascular Optical Coherence Tomography-Based Management in Plaque Erosion): A 1-Year Follow-Up Report. Circ Cardiovasc Interv 2017;10.

15. Jia H, Abtahian F, Aguirre AD, et al. In vivo diagnosis of plaque erosion and calcified nodule in patients with acute coronary syndrome by intravascular optical coherence tomography. J Am Coll Cardiol 2013;62:1748-58.

16. Hayes SN, Kim ESH, Saw J, et al. Spontaneous Coronary Artery Dissection: Current State of the Science: A Scientific Statement From the American Heart Association. Circulation 2018;137:e523-e57.

17. Cereda AF, Canova PA, Soriano FS. Spontaneous Coronary Artery Dissection After Pregnancy as First
Manifestation of a Vascular Ehlers-Danlos Syndrome. J Invasive Cardiol 2017;29:E67-8.

18. Finet G, Gilard M, Perrenot B, et al. Fractal geometry of arterial coronary bifurcations: a quantitative coronary angiography and intravascular ultrasound analysis. EuroIntervention 2008;3:490-8.

19. McPherson JA, Maehara A, Weisz G, et al. Residual plaque burden in patients with acute coronary syndromes after successful percutaneous coronary intervention. JACC Cardiovasc Imaging 2012;5:S76-85.

20. Guillon B, Moris M, Besutti M, et al. Evaluation of the EAPCI OCT criteria for optimization of angioplasty in the DOCTORS study population [Abstract]. Eur Heart J 2019;40:3090.

21. Prati F, Romagnoli E, Burzotta F, et al. Clinical Impact of OCT Findings During PCI: The CLI-OPCI II Study. JACC Cardiovasc Imaging 2015;8:1297-305.

22. Adriaenssens T, Joner M, Godschalk TC, et al. Optical Coherence Tomography Findings in Patients With Coronary Stent Thrombosis: A Report of the PRESTIGE Consortium (Prevention of Late Stent Thrombosis by an Interdisciplinary Global European Effort). Circulation 2017;136:1007-21.

23. Souteyrand G, Amabile N, Mangin L, et al. Mechanisms of stent thrombosis analysed by optical coherence tomography: insights from the national PESTO French registry. Eur Heart J 2016;37:1208-16.

24. Wolfrum M, De Maria GL, Benenati S, et al. What are the causes of a suboptimal FFR after coronary stent deployment? Insights from a consecutive series using OCT imaging. EuroIntervention 2018;14:e1324-31.

25. Im E, Kim BK, Ko YG, et al. Incidences, predictors, and clinical outcomes of acute and late stent malapposition detected by optical coherence tomography after drugeluting stent implantation. Circ Cardiovasc Interv 2014;7:88-96.

26. Qiu F, Mintz GS, Witzenbichler B, et al. Prevalence and Clinical Impact of Tissue Protrusion After Stent Implantation: An ADAPT-DES Intravascular Ultrasound Substudy. JACC Cardiovasc Interv 2016;9:1499-507.

27. Soeda T, Uemura S, Park SJ, et al. Incidence and Clinical Significance of Poststent Optical Coherence Tomography Findings: One-Year Follow-Up Study From a Multicenter Registry. Circulation 2015;132:1020-9.

28. Chamie D, Bezerra HG, Attizzani GF, et al. Incidence, predictors, morphological characteristics, and clinical outcomes of stent edge dissections detected by optical coherence tomography. JACC Cardiovasc Interv 
2013;6:800-13.

29. Liu J, Maehara A, Mintz GS, et al. An integrated TAXUS $\mathrm{IV}, \mathrm{V}$, and VI intravascular ultrasound analysis of the predictors of edge restenosis after bare metal or paclitaxeleluting stents. Am J Cardiol 2009;103:501-6.

30. Alegria-Barrero E, Foin N, Chan PH, et al. Optical coherence tomography for guidance of distal cell recrossing in bifurcation stenting: choosing the right cell matters. EuroIntervention 2012;8:205-13.

31. Park SJ, Ahn JM, Kang SJ, et al. Intravascular ultrasoundderived minimal lumen area criteria for functionally significant left main coronary artery stenosis. JACC Cardiovasc Interv 2014;7:868-74.

Cite this article as: Guillon B, Tauzin H, Ecarnot F, Meneveau N. How can optical coherence tomography be used to optimize percutaneous coronary intervention? Cardiovasc Diagn Ther 2020;10(5):1415-1428. doi: 10.21037/cdt.2020.04.02
32. Dato I, Burzotta F, Trani C, et al. Optical coherence tomography guidance for the management of angiographically intermediate left main bifurcation lesions: Early clinical experience. Int J Cardiol 2017;248:108-13.

33. Yamaji K, Ueki Y, Souteyrand G, et al. Mechanisms of Very Late Bioresorbable Scaffold Thrombosis: The INVEST Registry. J Am Coll Cardiol 2017;70:2330-44.

34. Huang J, Emori H, Ding D, et al. Comparison of Diagnostic Performance of Intracoronary Optical Coherence Tomography-based and Angiography-based Fractional Flow Reserve for Evaluation of Coronary Stenosis. EuroIntervention 2020;16:568-76. 